CAHIERS DE

NARRATOLOGIE

\section{Cahiers de Narratologie}

Analyse et théorie narratives

$38 \mid 2020$

Lusor in Fabula. Jeu vidéo et nouvelles frontières du récit

\title{
Valeur narrative des mécaniques ludiques et corporéité du joueur
}

Hélène Sellier

\section{OpenEdition}

\section{Journals}

Electronic version

URL: http://journals.openedition.org/narratologie/11692

DOI: 10.4000/narratologie.11692

ISSN: 1765-307X

Publisher

LIRCES

Electronic reference

Hélène Sellier, "Valeur narrative des mécaniques ludiques et corporéité du joueur", Cahiers de

Narratologie [Online], 38 | 2020, Online since 19 January 2021, connection on 29 January 2021. URL:

http://journals.openedition.org/narratologie/11692 ; DOl: https://doi.org/10.4000/narratologie.11692

This text was automatically generated on 29 January 2021.

Article L.111-1 du Code de la propriété intellectuelle. 


\title{
Valeur narrative des mécaniques ludiques et corporéité du joueur
}

\author{
Hélène Sellier
}

\section{Introduction}

1 La narrativité du jeu vidéo est souvent envisagée dans son rapport aux éléments ludiques et pensée à partir du rôle du joueur dans la construction du récit. Cette conception est au cœur de la recherche sur le jeu vidéo puisque la pseudo-controverse entre narratologie et ludologie ainsi que l'opposition entre Game Studies et Play Studies ont permis la structuration du champ scientifique ${ }^{1}$. Lorsque Aarseth définit en 1999 le jeu vidéo comme forme de littérature ergodique qui, par sa non-linéarité demande un effort non-trivial au lecteur, il oriente les discussions dans cette voie. À sa suite, Britta Neitzel ${ }^{2}$, Marie-Laure Ryan ${ }^{3}$ ou encore Dominic Arsenault ${ }^{4}$ tentent de définir les modalités particulières du récit vidéoludique. Ce dernier soutient qu'il existe ainsi « un récit enchâssé invariable et prédéfini, que l'on peut extraire du jeu-objet pour l'analyser selon une approche formaliste ou structuraliste, et un récit vidéoludique variable à l'intérieur des contraintes de l'algorithme, qui prend vie dans le jeu manifeste et qu'il faut nécessairement observer à travers une approche centrée sur l'expérience. $»^{5}$ Cette dualité se retrouve dans le modèle de Rémi Cayatte qui explique que « le jeu vidéo raconte en instaurant un dialogue quasi-simultané entre les auteurs d'une expérience-cadre, et les joueurs, "auteurs » d'une ou plusieurs procédures de jeu $^{6} »$. La notion d'expérience-cadre, inspirée des travaux de Sélim Krichane ${ }^{7}$, fait écho aux travaux qui questionnent la diversité des formes narratives au sein de la structure de jeu, notamment aux propositions de Jenkins ${ }^{8}$. Ce dernier distingue, à partir d'une appréhension des récits vidéoludiques basée sur l'espace, les lieux évocateurs, les histoires jouées, les récits enchâssés et les récits émergents. Fanny Barnabé étudie plus largement les "éléments de narrativisation» des jeux vidéo qui comprennent par exemple les textes ou les angles de vue, mais aussi les objectifs et les règles du jeu' Sébastien Genvo, dont les travaux portent sur l'expressivité du jeu vidéo ${ }^{10}$, avait déjà souligné la nécessité de comprendre ensemble le système ludique et la portée narrative 
du jeu en analysant Passage de Jason Roher ${ }^{11}$. Dans une double démarche d'analyse et de création, Vincent Mauger propose le modèle EST (éléments, schémas, transversaux) qui permet de lier les pratiques traditionnelles de scénarisation, de construction de monde et de game design ${ }^{12}$. Redéployant les problématiques originelles du champ concernant la narrativité vidéoludique, les recherches actuelles invitent ainsi à penser le jeu vidéo comme un ensemble.

2 Cette conception du jeu vidéo est favorisée par les développements de l'industrie culturelle à partir des années 2010. Parallèlement à la vague des jeux indépendants, qui, créés par des équipes réduites, s'éloignent des codes des productions à gros budget et tentent de se démarquer par leur originalité, la production est marquée par la popularisation du terme narrative designer (plutôt que game writer ou scénariste). Créé en 1997 par Madison Smartt Bell, le terme narrative design est régulièrement utilisé depuis les années 2010 dans les studios de jeux vidéo pour désigner l'appréhension de la narrativité vidéoludique non seulement par le texte mais aussi par les autres systèmes sémiotiques. Le manuel de référence dirigé par Wendy Despain, Professional Techniques for Video Game Writing, contient un article de Jay Posey offrant une première définition de la pratique créatrice :

3 Si le récit est "l'histoire que notre jeu raconte", le narrative design est la création de cette histoire et le design des mécaniques par lesquels elle est raconté. C'est-à-dire que le narrative design comprend non seulement l'histoire en elle-même mais aussi la manière dont l'histoire est communiquée aux joueurs et dont les autres éléments du jeu accueillent et immergent le joueur dans le monde du jeu ${ }^{13}$.

4 Les descriptions plus récentes insistent aussi sur l'hybridité du travail. Heussler et ses collègues écrivent ainsi qu'un narrative designer est «le défenseur de l'histoire interactive » auprès de l'ensemble de l'équipe et qu'il « combine les rôles du scénariste avec ceux du game designer ${ }^{14} »$. Si le poste est souvent défini à la jonction entre l'écriture de textes et la conception de mécaniques, les définitions s'ouvrent aussi aux autres corps de métier (level design, game design, UX, sound design, localisation...) :

5 Le game narrative design est l'art de raconter une histoire dans un jeu vidéo en utilisant les techniques et les moyens disponibles. C'est l'art d'utiliser le gameplay et la somme des méthodes visuelles et acoustiques pour créer une expérience divertissante et engageante pour les joueurs ${ }^{15}$.

6 À la suite des succès de jeux narratifs comme Heavy Rain (Quantic Dream, 2010), Dear Esther (The Chinese Room, 2012) The Walking Dead (Telltale Games, 2012), Gone Home (Fullbright, 2013), Life is Strange (Dontnod, 2015), émergent des logiques de production qui cherchent à mettre au centre du processus de création la question de la narrativité en redéfinissant la répartition des tâches et en tentant d'inventer de nouvelles méthodes de conception.

7 Cet article s'inscrit au cœur de ces préoccupations actuelles en questionnant les matérialisations de la narrativité vidéoludique. En effet, si les travaux au sein du champ académique comme les discours au sein de l'industrie culturelle pointent vers l'harmonie potentielle de la structure vidéoludique dans la création d'une expérience cohérente, les recherches sur ce sujet sont encore à leurs débuts. Dans le premier domaine, je renverrai par exemple aux travaux que Rémi Cayatte a mené au sein de l'Expressive Game Lab en partenariat avec Goblinz Studios ${ }^{16}$. Dans le second champ, il est possible de penser aux interrogations qu'Emily Short, autrice de fiction interactive 
reconnue dans le milieu anglo-saxon, mène à l'heure actuelle sur son $\operatorname{blog}^{17}$. Le sommaire du récent livre The Advanced Game Narrative Toolbox, qui se présente comme un complément au manuel de 2015, montre aussi la difficulté de mettre en mot les pratiques naissantes et de se détacher des problématiques habituellement traitées dans les ouvrages de scénarisation (comme la question des représentations, les formats et genres, les outils, les modalités pratiques... ${ }^{18}$. De manière générale, par habitude, on persiste encore à distinguer la structure narrative et la structure ludique ainsi qu'à penser d'un côté, le jeu comme objet et de l'autre, le jeu comme activité. Plutôt que de proposer un nouveau modèle, comme le fait Esteban Giner $^{19}$, il est aussi possible de garder ces dichotomies dans l'analyse, c'est-à-dire de penser les œuvres en prenant en compte le discours social qui accompagne la production et la réception des jeux vidéo. $\mathrm{Au}$ lieu de se baser sur une définition du récit en fonction du temps ${ }^{20}$ ou en fonction de l'espace ${ }^{21}$, cet article emprunte à la narratologie post-classique -- dont l'ambition d'être " plus hospitalière ", " plus modeste ", " plus utilitaire $»^{22}$ correspond au projet-- une conception de la narrativité comme processus intellectuel et émotionnel plutôt que comme produit ${ }^{23}$, notamment dans la lignée des travaux de Raphaël Baroni ${ }^{24}$. Parallèlement, la définition des mécaniques ludiques suit les travaux de Aki Järvinen ${ }^{25}$, Miguel Sicart ${ }^{26}$ et Damien Hansen ${ }^{27}$ qui les distinguent des règles. Pour les trois auteurs, elles se définissent comme des moyens qui influencent les actions du joueur en contraignant l'espace des possibles pour atteindre des objectifs. Ce sont des « attributs qui sont codés de façon constante, immuable et déterminée à l'avance ${ }^{28} »$. Le concept permet donc de saisir le fonctionnement du logiciel informatique, mais il pose aussi en creux la question des usages avoués, dissimulés ou imprévus. Comme la perspective cognitive de la narrativité, il permet de faire coïncider une approche du jeu vidéo comme objet et comme activité.

8 Cet article cherche en effet à poursuivre les interrogations sur la proximité entre ludique et narratif dans la construction du sens de l'expérience vidéoludique. Y est défendue l'idée d'une valeur narrative de l'expérience corporelle des mécaniques ludiques. Son objectif est limité : ouvrir le dialogue autour de trois pistes de réflexion qui nécessiteront d'être développées, retravaillées, critiquées. À partir d'un ensemble de travaux orientés vers des problématiques de design, seront d'abord abordées les problématiques autour des concepts de dissonance ludo-narrative et de mécanique narrative. Ensuite, des recherches sur la gestualité dans le cadre du numérique permettront de continuer la réflexion en soulignant l'importance de la corporéité dans l'expérience narrative du jeu vidéo.

9 Le travail a été mené dans une perspective de recherche-création ${ }^{29}$, et plus particulièrement sous deux modes décrits par Owen Chapman et Kim Sawchuck ${ }^{30}$. D'abord, la recherche est conçue comme socle de la création, dans la mesure où les théories et les analyses d'œuvres proposées dans cet article fournissent une base pour la pratique. Ensuite, la recherche est formée à partir de la création. Cette dernière permet alors de tester des hypothèses et son analyse fournit des résultats. Le travail a été mené à travers différents projets et cet article se concentrera sur le jeu vidéo RecovR (The Seed Crew, à paraître) ${ }^{31}$, qui est à la fois le fruit des recherches précédentes et le terreau de nouvelles expérimentations. Le premier module porte sur le sexisme en entreprise et est constitué d'une série d'épisodes dans lesquels Charlie, l'avatar du joueur, se déplace dans les bureaux et discute avec ses collègues. Chaque épisode est 
ponctué par un combat que Charlie mène en son for intérieur pour lutter contre les agressions quotidiennes qu'elle subit.

\section{Dissonance et harmonie ludo-narrative}

$10 \mathrm{Au}$ sein de l'industrie du jeu vidéo, la question du lien entre narratif et ludique s'est cristallisée dans la notion de dissonance ludo-narrative qui a émergée en 2007 dans une critique du jeu Bioshock (2K Games, 2007) écrite par Clint Hocking ${ }^{32}$, qui a notamment travaillé comme directeur créatif chez Lucas Arts et Ubisoft. Le jeu met en scène deux philosophies, une approche empathique et une approche rationaliste, qui sont souvent évoquées à travers un élément particulier du jeu, le choix qu'a le joueur de "sauver " les «petites sœurs» ou de les "récolter» afin d'obtenir un bénéfice immédiat. Pour Hocking, «Bioshock souffre d'une puissante dissonance entre ce dont il parle en tant que jeu et ce dont il parle en tant qu'histoire ${ }^{33} »$. Alors que les mécaniques de jeu laissent le choix au joueur d'adopter l'une des deux philosophies, la structure narrative (ou le récit enchâssé, pour reprendre la terminologie d'Arsenault) l'oblige à aider Atlas qui incarne l'opposition à l'objectivisme. Hocking critique ce fonctionnement comme inadéquat pour le joueur, en le jugeant irrespectueux. À partir de cette analyse de cas, la notion de dissonance ludo-narrative a été l'objet de nombreuses discussions dans la sphère des créateurs de jeux vidéo. Elle est souvent exemplifiée par la contradiction flagrante entre le récit d'un héros bienveillant et le gameplay qui consiste à tuer des ennemis, comme le fait par exemple Daniel Dunne ${ }^{34}$. Dans son article, ce dernier encourage les concepteurs de jeux vidéo à prendre exemple sur les créateurs de romans graphiques en considérant la manière dont les différents modes peuvent contribuer ensemble à l'expression d'un unique message.

11 Le succès du concept de dissonance ludo-narrative s'explique non seulement parce qu'il permet de penser le lien entre des disciplines habituellement distinctes, au sein des studios de jeux et plus largement au sein des industries culturelles, mais aussi parce qu'il met au centre de la réflexion l'attitude du joueur. En lien avec l'idéalisation de l'immersion comme l'état de jeu parfait (que repèrent Fanny Barnabé et Julie Delbouille ${ }^{35}$ ), le phénomène a largement été discuté comme un problème, une erreur de design, dans la mesure où il décrit une distance entre le joueur et le jeu ${ }^{36}$. Brett Makedonski, scénariste indépendant, la présente par exemple comme une contradiction logique : «La dissonance ludo-narrative est l'idée selon laquelle, lorsqu'un jeu raconte quelque chose par son histoire et son environnement puis se contredit à travers son gameplay, le joueur perd son immersion et se déconnecte en partie de l'expérience ${ }^{37}$.» Une approche similaire est adoptée par Toh Wiemin qui s'appuie, dans son travail de thèse, sur les travaux en narratologie cognitive de Monika Fludernik ${ }^{38}$ et Marie-Laure Ryan ${ }^{39}$. Définir le récit vidéoludique comme l'interprétation du joueur de son interaction avec le jeu lui permet de distinguer trois types de relations entre le récit et le gameplay, qui dépendent de la réception : la dissonance ludonarrative, l'insignifiance ludo-narrative (lorsque les deux structures ne sont pas envisagées de façon concomitante) et la résonance ludo-narrative. Cette dernière " arrive lorsque les concepts narratifs et de gameplay sont tellement intégrés qu'ils ne peuvent pas être séparés " et donne lieu à une "expérience émotionnelle intensifiée $e^{40} \%$. Des conclusions similaires sont tirées par les créateurs qui cherchent alors à créer des expériences ludo-narratives cohérentes. Wendy Despain et Lauryn 
$\mathrm{Ash}^{41}$ établissent par exemple un modèle de design pour créer une harmonie ludonarrative en pensant en relation les mécaniques de jeu, le récit, le contexte et les émotions du joueur.

Dans cette quête d'une cohérence ludo-narrative, le degré zéro de la valeur narrative des mécaniques ludiques consiste peut-être à faire dépendre un récit enchâssé de la manière dont le joueur joue. Dans Oxenfree (Night School Studio, 2016), le joueur a le choix entre plusieurs options de dialogues ou rester silencieux, comme de nombreux autres jeux, mais il peut aussi s'éloigner du lieu de la conversation pour ramasser un objet ou découvrir un lieu sans que la discussion ne s'interrompe. L'ensemble des éléments du récit n'est alors pas connu. Dans Lone Survivor (Superflat Games, 2012), l'usage des mécaniques ludiques conditionne le récit enchâssé de manière plus radicale : la fin est déterminée par l'utilisation des mécaniques par le joueur, comme le nombre d'ennemis tués ou la nourriture consommée pendant sa partie. Dans le projet de recherche-création RecovR, la création de liens entre les différents moments de jeu (exploration des bureaux, dialogues, phase de combat) est un objectif de design qui a été fixé dès la phase de conception. Les quatre postures de combat correspondent ainsi aux quatre options de dialogue qui ont été construites pour représenter différents comportements de confrontation et d'évitement face au sexisme ${ }^{42}$. L'utilisation des postures, plus ou moins intensive, détermine les choix qui sont ensuite accessibles dans les conversations. À l'inverse, la manière dont le joueur réagit dans les discussions qui précèdent le combat influence le niveau, au début de cette phase, de la mécanique qui s'apparente à la «barre de vie » et qui représente la confiance en soi de Charlie. Les mécaniques ludiques dépendent dans ce cas du récit enchâssé comme c'est le cas dans d'autres jeux. Star Wars: Knights of the Old Republic II - The Sith Lords (Obsidian Entertainment, 2004) détermine par exemple les classes de prestige auxquelles peut avoir accès le joueur en fonction de son alignement (au côté clair ou au côté obscur), principalement déterminé par des choix de dialogue.

L'élaboration de liens entre mécaniques ludiques et récits enchâssés est une méthode créative qui vise une expérience de jeu harmonieuse. Cependant, la dissonance ludonarrative n'est pas nécessairement une erreur de design, tout comme l'immersion n'est pas une posture ludique plus commune ou plus appropriée que la réflexivitét ${ }^{43}$. Nick Ballantyne compare la dissonance ludo-narrative à la dissonance cognitive ${ }^{44}$. Dans le cas de la seconde, l'écart entre les croyances et les actions peut être résolu de plusieurs manières (modification du système de valeur, actions différentes, refus de le prendre en compte...). Au contraire, la dissonance ludo-narrative correspond au fait que le joueur se voit imposer un système de valeurs sans qu'il ait la possibilité de réagir : «s'il ne nous est pas possible [en tant que joueur] de changer notre manière de jouer ou d'ignorer les contradictions, nous n'avons pas d'autres choix que de justifier la dissonance ${ }^{45}$. » Puisqu'elle crée une distance entre le joueur et le jeu, la dissonance ludo-narrative permet, pour Ballantyne, d'inciter le joueur à la réflexion. S'appuyant sur la réflexion de Ballantyne, Frédéric Séraphine soutient qu'il existe un potentiel émotionnel de la dissonance ludo-narrative qui tient à «l'émersion» du joueur, «la sensation d'être tiré en dehors de l'expérience de jeu ${ }^{46} »$. Séraphine choisit d'illustrer cette idée en évoquant les monstres de Silent Hill 2 qui ne peuvent pas être tués et symbolisent ainsi la culpabilité. Un exemple qui insiste davantage sur l'utilisation par le joueur des mécaniques ludiques peut être pris dans Nier (Cavia, 2010). Le joueur est invité à compléter plusieurs fois le jeu et chaque nouvelle actualisation donne lieu à l'apparition de nouveaux éléments au sein du récit enchâssé. Ainsi, en jouant pour la 
deuxième fois, le joueur peut comprendre les discours des ennemis qu'il affronte. Cette humanisation lui laisse entendre que les monstres qu'il jugeait mauvais ne sont que des êtres qui tentent de survivre. Pourtant, s'il veut progresser dans le jeu, il doit malgré tout continuer le combat. Ce dispositif, cette tension entre mécaniques ludiques et récit enchâssé, permet à Yoko Taro, à qui est attribuée la paternité du jeu, de questionner ce que signifie être humain. Le sens de l'expérience narrative est construit à l'interstice entre les mécaniques et le récit enchâssé. The Last Guardian (SIE Japan Studio and GenDesign, 2015) utilise un fonctionnement similaire à un moment précis. Une des mécaniques de jeu récurrente consiste à briser des yeux de verre : l'enfant, contrôlé par le joueur, permet ainsi à son compagnon, la bête nommée Trico, d'avancer, puisqu'il en a peur. Cependant, au sein d'une cinématique, lorsque l'enfant est capturé, Trico vient le sauver malgré la présence d'un œil. Le récit enchâssé n'est pas cohérent avec les mécaniques ludiques mais cette dissonance ludo-narrative permet d'illustrer l'amitié entre les deux êtres. De même, le projet de recherche-création RecovR utilise des moments de disjonction pour leur potentiel émotionnel et réflexif. La mécanique ludique principale consiste en l'identification d'une remarque ou une situation sexiste au sein des conversations. Puisque le bouton est explicitement placé en dehors de la diégèse, la reconnaissance n'est pas attribuée à Charlie, l'avatar, mais au joueur. Cette caractéristique du jeu a été conçue pour tester l'hypothèse suivante. La mécanique matérialise non seulement la relation complexe à l'avatar, qui n'est pas uniquement de l'ordre de l'identification (sentiment de devenir l'avatar) mais aussi de l'empathie ou de la sympathie ${ }^{47}$, mais elle favorise aussi une attitude réflexive pour le joueur, dans la mesure où Charlie, comme les autres personnages, peut avoir des commentaires inappropriés. Le jeu suppose que le sens se construit dans le rapport contradictoire entre le récit enchâssé (remarque sexiste) et la mécanique ludique (indication du sexisme) grâce au rapport à l'avatar : puisque le sexisme est ancré dans les codes culturels, tout le monde est susceptible de faire des erreurs, d'être blessant sans le vouloir.

14 S'interroger sur les effets de formes de dissonance et d'harmonie entre récit enchâssé et possibilité d'action du joueur constitue une première étape pour comprendre la portée narrative des mécaniques ludiques. Cependant, les préoccupations récentes concernant d'autres modèles narratifs témoignent d'une évolution des débats. Frédéric Séraphine montre ainsi qu'une solution créative souvent évoquée en réponse au problème de la dissonance ludo-narrative consiste en « l'annihilation de toute forme de structure narrative pré-établie ${ }^{48}$ ». Elle revient à privilégier des formes de récits émergents, qui laissent au joueur la charge de la construction du récit, aux modes de récits enchâssés (comme les cinématiques, les dialogues...). Puisque Minecraft (Mojang, 2011) propose d'explorer et de construire des univers toujours différents et autorise les interactions entre joueurs, il est souvent cité pour exemplifier la manière dont les événements $d u$ monde ludique construit par les joueurs sont perçus comme une histoire. La fin des années 2000 marque l'émergence de la notion de récit émergent, créée par Henri Jenkins ${ }^{49}$, dans les discours des créateurs. Par exemple, Sandy Louchart, Ruth Aylett, Joäo Dias et Rui Figureiredo (2008) expliquent leur méthode de création pour développer des récits émergents basés sur les interactions entre personnages ${ }^{50}$. Simon Chauvin, Guillaume Levieux, Jean-Yves Donnart et Stephane Natkin proposent en 2014 une autre méthodologie en déterminant cinq caractéristiques principales : la cohérence, l'agentivité, l'espace de possibilité, l'incertitude et le statut de co-auteur ${ }^{51}$. À travers la notion de récit émergent, les créations et réflexions contemporaines mettent 
plus largement l'accent sur la valeur narrative qu'ont en elles-mêmes les mécaniques ludiques.

\section{Mécaniques narratives}

15 L'idéal, encore largement partagé au sein de la communauté des créateurs, d'une harmonie des systèmes sémiotiques au sein de l'expérience vidéoludique est le fondement d'observations sur les mécaniques dites narratives. Teun Dubbelman soutient que "les mécaniques narratives de jeu invitent les agents, notamment le joueur, à réaliser des actions qui supportent la construction d'histoires immersives et de mondes fictionnels dans l'esprit incarné du joueur ${ }^{52}$. " Il détaille dans son article trois exemples de mécaniques qui permettent de créer des événements fictionnels intéressants. En analysant Left 4 Dead, il décrit d'abord « une utilisation de l'espace qui crée des tensions ", renvoyant indirectement aux récits émergents qui, on l'a déjà dit, ont été proposés comme solution à la dissonance ludo-narrative. L'étude du combat au pistolet à eau de The Last of Us: Left Behind le conduit ensuite à identifier comme mécanique narrative la recontextualisation des mécaniques ludiques afin de créer l'empathie. Finalement, il étudie Papers, please (3909 LLC, 2013), jeu dans lequel le joueur incarne un agent de l'immigration chargé de contrôler les documents pour autoriser ou non l'entrée dans le pays. L'œuvre est utilisée pour montrer comment des mécaniques ludiques peuvent créer des récits de dilemmes moraux : "Selon la manière dont le joueur utilise les mécaniques et les règles [c'est-à-dire s'il respecte scrupuleusement les lois ou non], plusieurs histoires peuvent se dérouler ${ }^{53}$ ", notamment concernant la famille du protagoniste qui dépend de lui. Comme Dubbelman, les créateurs et les chercheurs font souvent appel au jeu de Pope au sein des réflexions sur les liens entre narratif et ludique. Plus particulièrement, l'œuvre sert à questionner la manière dont la structure ludo-narrative peut engager le joueur dans une réflexion éthique ${ }^{54}$.

Bjarke Larsen et Henrik Shoenau-Fog s'en servent afin de démontrer l'idée d'une qualité narrative des mécaniques ludiques ${ }^{55}$. Dans Papers, please, selon eux, «à travers les mécaniques, les joueurs eux-mêmes ont l'occasion de "sentir" le récit et de faire l'expérience de leur propre version de l'histoire qui transparaît à travers le gameplay et le contexte ${ }^{56}$ " (qui regroupe à la fois le récit enchâssé et sa présentation par les graphismes et les sons). Larsen et Shoenau-Fog soutiennent alors que «les mécaniques ludiques communiquent intrinsèquement un récit à travers les possibilités et la valorisation des actions données au joueur ${ }^{57}$ ». Dans Loneliness de Jordan Magnuson (2011), le joueur contrôle un carré noir et d'autres formes identiques bougent à l'écran, par groupes. Dès que le carré contrôlé par le joueur s'approche des autres, ceux-ci s'éloignent. Les possibilités d'action données au joueur sont réduites : en déplaçant le curseur dans l'espace virtuel, il peut soit essayer d'éviter les autres éléments, soit les voir continuellement s'écarter. Quel que soit son choix, le jeu donne une expérience de la solitude. James Portnow forme en 2012 l'expression «mécaniques comme métaphores » à partir de l'analyse de ce jeu ${ }^{58}$.

17 À l'origine de cette idée selon laquelle le système ludique peut raconter une histoire, se trouve une conception de design couramment partagée au sein de l'industrie : les mécaniques sont des abstractions d'action et peuvent être identifiées par des verbes ${ }^{59}$. Dans Game Design Vocabulary de Anna Anthropy et Naomi Clark (2014), on trouve une analyse d'un jeu qui va dans ce sens. Dans The Best Amendment de Paolo Pedercini (2013) 
les ennemis qu'affrontent le joueur avec une arme à feu, représentées par des silhouettes noires, sont des enregistrements de l'avatar $\mathrm{du}$ joueur réalisés précédemment au cours de la partie. Selon Anthropy et Clark, «The Best Amendment n'explique pas son message avec des mots mais en créant un système qui produit certains types d'expériences lorsqu'il est joué, Pedercini fait passer une idée : le gentil avec une arme à feu dépend de nos perceptions et se promener en tirant sur des soidisant "méchants" mène à un massacre ${ }^{60}$ ». Plus récemment, Emily Short explique que son processus d'écriture commence par l'identification des actions qui sont possibles dans le jeu. Elle évoque par exemple « la préparation qui consiste à trouver une bonne mécanique pour le narrative design ${ }^{61} »$ dans sa lecture de The Anatomy of a story de John Truby où elle évoque Papers, please. L'identification des abstractions d'action adéquates par rapport à l'histoire envisagée va de pair avec la conception des modalités d'emploi de la mécanique. Séraphine prend l'exemple de The Last of Us (Naughty Dog, 2013) pour montrer que «changer les mécaniques ludiques ou les faire disparaître peut parfois avoir un sens concernant le monde du jeu ou ses personnages ${ }^{62}$. " Dans le jeu de Naughty Dog, l'avatar du joueur peut appeler Ellie afin de la porter pour qu'elle ouvre la voie. La disparition de cette mécanique alors que le bouton est encore actionnable exprime le fait qu'Ellie soit émotionnellement blessée. Dans la même logique, au sein du projet de recherche-création RecovR, le combat a été l'occasion de tester l'implémentation d'une mécanique narrative qui fonctionne par rapport aux attentes du joueur : refuser de se battre ou échouer à vaincre les ennemis lors du combat ne signifie pas que le jeu s'arrête ou que le joueur ait l'obligation de recommencer. Si la barre de vie, représentant la confiance en soi de Charlie, arrive à zéro, la conversation se poursuit sous des modalités particulières, exprimant l'idée que, quel que soit notre état émotionnel ou notre capacité à gérer une situation, les événements se déroulent sans avoir la possibilité d'y échapper.

Les réflexions actuelles sur les mécaniques narratives qui alimentent le travail de recherche- création doivent aussi être mise en relation avec la fortune du concept de rhétorique procédurale créé par Ian Bogost ${ }^{63}$. Ce dernier affirme que les jeux vidéo sont "persuasifs », c'est-à-dire qu'ils utilisent une nouvelle forme rhétorique qui dépend leur fonctionnement procédural et transmettent aux joueurs des messages prédéfinis sur les systèmes culturels, politiques, économiques, religieux.... S'appuyant sur la thèse de Bogost, les travaux sur l'«expressive processing» de Noah Wardrip-Fruin développent cette ligne de pensée. L'auteur définit deux axes majeurs que recouvre le concept : «l'expression des auteurs et ce que les processus informatiques signifient, sans que cela soit nécessairement visible par les publics ${ }^{64}$ ». Dans son article "Against Procedurality ", Miguel Sicart montre ainsi à juste titre que les game designers s'appuient sur le concept de rhétorique procédurale à la fois pour justifier leur approche d'auteur et pour montrer que les jeux ont une valeur artistique ${ }^{65}$. Sans remettre en cause complètement la pertinence d'une approche formaliste, Sicart soutient au contraire que cette manière de penser les jeux vidéo ne prend pas en compte l'importance du jeu au sens de play.

\section{Corporéité de l'expérience narrative}

Les travaux de Sicart, et plus largement la dynamique insufflée par les Play Studies, invitent à davantage considérer l'activité de jeu dans les expérimentations sur la valeur 
narrative des mécaniques ludiques. Plus particulièrement, il est possible de poursuivre les réflexions menées à partir des concepts de dissonance ludo-narrative et de mécanique narrative en intégrant une interrogation sur la manière dont le geste du joueur participe à la construction du sens de l'expérience. Thomas Morisset propose de penser le jeu vidéo comme " un art du geste utilisant l'image " ${ }^{66}$ et, dans le cadre d'un travail sur la narrativité vidéoludique, il est possible de s'interroger sur la portée narrative des mouvements du joueur.

Dans le cadre de ses recherches sur la littérature numérique, Serge Bouchardon montre qu'au sein des créations numériques, le geste a un rôle particulier :

Pour pouvoir analyser la dimension manipulable dans les créations numériques, il s'agit ainsi de penser le geste ni uniquement à partir du geste en soi, ni uniquement à partir de ce qui se passe à l'écran. Dans la manipulation, le geste prend sens dans l'interaction ${ }^{67}$.

Un extrait de The Wolf Among Us (Telltale Studios, 2013) illustre parfaitement le rôle du geste en lien avec l'image vidéoludique dans l'élaboration du sens. Le joueur incarne un shérif qui enquête sur un meurtre avec des méthodes plus ou moins violentes selon les choix effectués. À un moment, il doit décider d'arrêter le combat ou d'arracher le bras d'une créature qui a défié l'avatar. En choisissant la seconde option, le joueur s'attend à ce qu'une cinématique se déroule. Au contraire, le jeu lui demande de presser à répétition sur la touche $Q$ pour que l'action se réalise. Le jeu utilise un QTE mais, contrairement à l'usage courant de la mécanique, il ne s'agit pas de faire l'action dans un temps donné. C'est la concordance entre la répétitivité de l'action et la représentation du bras qui progressivement est arraché qui donne le sens à la scène. Le geste permet de faire ressentir au joueur le poids de ses décisions et participe largement à la construction de l'effet d'horreur.

23 La gestualité du jeu vidéo est souvent comprise comme un rythme dont le joueur ferait l'expérience grâce à son corps. Järvinen parle de « clickability " ${ }^{68}$ pour définir le plaisir de cliquer en rythme, quel que soit le résultat dans le jeu et propose de prendre en compte cette activité ludique dans la création de jeux sociaux en ligne. Replaçant au centre du concept l'idée d'une évaluation par le programme, Bruno Dupont définit plus largement le rythme vidéoludique comme «la réalisation par le joueur, dans un ordre variable, d'une suite de mouvements-types donnés à une vitesse donnée, dans le but d'arriver à un résultat considéré comme positif par le gameplay ${ }^{69}$ ». En s'appuyant sur la conception du rythme de Deleuze et de Guattari, il montre que le rythme du jeu vidéo ne se fait pas en mesure, à intervalles réguliers, mais vient au contraire de la friction entre différentes actions répétées. La gestualité du jeu vidéo prendrait alors son sens dans les moments de disjonction, quand le rythme change. Dans Brothers: A tale of two sons (Starbreeze Studios, 2013), le joueur contrôle deux personnages, deux frères qui ont chacun des capacités différentes. Le grand frère est plus fort et peut activer des leviers tandis que le jeune frère peut se faufiler dans des passages étroits. Le premier est contrôlé avec la partie gauche de la manette tandis que le second est contrôlé avec la partie droite. Ce qui est inhabituel au départ pour un joueur expérimenté devient progressivement naturel au fur et à mesure du jeu. Un rythme particulier à ce jeu s'installe dans le corps du joueur. Cependant, à un moment, le grand frère est blessé. Le jeune frère va chercher de l'eau pour le sauver et c'est la première fois que le joueur n'utilise plus qu'une partie de sa manette. Le changement du rythme installé par le jeu donne pleinement son sens à l'expérience. Lorsque le jeune frère revient, le grand-frère est mort. L'aventure continue et le joueur, privé d'une partie des contrôles auxquels il 
est habitué, ressent physiquement un manque. Plus tard, se présente une rivière qu'il est impossible de traverser en utilisant simplement le côté droit de la manette. Ce n'est qu'en manipulant les deux joysticks que le personnage-joueur peut avancer. La représentation du souvenir d'un être décédé et le discours sur la construction de soi par la mémoire passe ici par le geste du joueur. C'est l'imbrication entre récit enchâssé, mécaniques de jeu et leurs actualisations dans des gestes aux rythmes irréguliers qui semble construire le sens narratif.

Le projet RecovR permet de mettre à l'épreuve cette idée. Dans la phase de combat, chaque posture, associée à une forme de réaction au sexisme, déclenche un mini-jeu au rythme particulier qui tente de traduire les comportements de confrontation et d'évitement par les mouvements. Dans son article «Towards Gestural Specificity in Digital Literature ", Serge Bouchardon présente les résultats d'un travail de recherchecréation et explique que son approche pour créer du sens grâce aux gestes consiste en un travail de défamiliarisation : «avec le numérique, le geste interactif et la manipulation gestuelle interactive sont défamiliarisés grâce à l'opacité du programme : le digital peut introduire un écart entre les attentes de l'utilisateur à partir de ses gestes et le champ des possibilités offert ${ }^{70}$. " Au contraire, RecovR, qui s'adresse à un large public, utilise des manipulations courantes au sein des productions numériques sans chercher à modifier leur interprétation. Pour évoquer des situations problématiques, dont le joueur n'a pas nécessairement l'habitude, il a été décidé de travailler des sensations communes et des gestes partagés. Cette approche nécessite de prendre en compte le corps comme une construction socio-culturelle. En suivant des réflexions menées à propos de la danse contemporaine, il semble alors préférable d'évoquer la corporétité, qui est "une entité affective, subjective et symbolique " plutôt que le corps, qui renvoie à une «entité stable et homogène $»^{71}$. Les rythmanalyses, menées par Matthieu Triclot ${ }^{72}$, ont formé un socle particulièrement utile pour penser la prise en main des contrôles pour créer des rythmes variés à partir de modèles existants. Comprenant les jeux vidéo comme des "expériences instrumentées ${ }^{73}$, Triclot s'intéresse en effet aux actualisations réelles du jeu dans le corps des joueurs en étudiant les entrées sur les contrôles du jeu selon différentes modalités : la vitesse de l'exécution (nombre de clics par seconde), sa régularité (écarttype de la durée entre les appuis) et son style (durée moyenne d'un appui). En étudiant des jeux s'inscrivant au sein de divers genres vidéoludiques, il distingue alors plusieurs registres d'action qui ont inspiré la création. Par exemple, la réaction passionnée de l'ordre d'un comportement de confrontation au sexisme est associée au geste consistant à un appui rapide et répété à un seul endroit, tandis que la réaction de conciliation de l'ordre d'un comportement d'évitement est liée à des appuis irréguliers à plusieurs endroits de l'écran. Dans le modèle proposé par Triclot, la première expérience est de l'ordre de «la frénésie » et la seconde de l'ordre de «la danse ». En adaptant au processus de création les modalités d'une étude qui porte sur les pratiques de jeu en situation, il a été décidé de «se focaliser sur la production gestuelle de la performance » en faisant "l'économie du recours à l'image écranique » dans la phase de conception. Les représentations visuelles associées aux gestes ont été établies dans un second temps (remplissage d'une jauge et éclatement de bulles pour les cas précédents). Le jeu suppose alors que l'engagement corporel du joueur en lui-même participe à la construction du sens de l'expérience. 


\section{Conclusion}

S'appuyant sur un travail d'appropriation de travaux de recherches et de l'étude formelle de plusieurs œuvres, le processus de création permet de mettre en œuvre des hypothèses concernant l'interdépendance des systèmes sémiotiques dans la narrativité vidéoludique. Cet article met plus précisément en lumière les potentiels expressifs de la dissonance et de l'harmonie ludo-narrative, la portée narrative des mécaniques ludiques et l'importance de la corporéité dans la construction du sens de l'expérience. Par la suite, le travail de narrative design réalisé devra être complété par des analyses des pratiques afin de tirer des conclusions sur la validité des idées proposées.

\section{BIBLIOGRAPHY}

Anthropy Anna et Clark Naomi, Game Design Vocabulary: Exploring the Fundational Principles Behind Good Game Design, Boston: Addison-Wesley Professional, 2014

Arsenault Dominic, « Jeux et enjeux du récit vidéoludique : la narration dans le jeu vidéo » [En ligne], Mémoire de master en études cinématographiques, sous la direction de Bernard

Perron, université de Montréal, 2006. URL : http://www.le-ludophile.com/Files/

Dominic\%20Arsenault\%20-\%20Memoire.pdf (consulté le 20/07/2020)

Audet René, «La narrativité est affaire d'événement », in Jeux et enjeux de la narrativité dans les pratiques contemporaines, Rene Audet et al., Paris : Dis Voir, 2006.

Ballantyne Nick, « The Why, What and WTF: Ludonarrative Dissonance », Gamecloud [En ligne], 15 février 2015. URL : https://gamecloud.net.au/features/opinion/twwwtf-ludonarrativedissonance (consulté le 20/07/2020)

Barnabé Fanny, Narration et jeu vidéo. Pour une exploration des univers fictionnels, mémoire de Master, sous la direction de Bjorn-Olav Dozo, université de Liège, 2012.

Barnabé Fanny et Delbouille Julie, « Aux frontières de la fiction : l'avatar comme opérateur de réflexivité », Sciences du jeu [En ligne], n 9, 2018. URL : http://journals.openedition.org/sdj/ 958 (consulté le 20/07/2020)

Baroni Rapahël, Les Rouages de l'intrigue. Les outils de la narratologie postclassique pour l'analyse des textes littéraires, Genève, Slatkine, 2017.

Bogost Ian, Persuasive Games, Cambridge \& London: The MIT Press, 2007.

Bouchardon Serge, « Des figures de manipulation dans la création numérique », Protée, vol. 39, n 1, 2011, pp. 37-46. URL : https://doi.org/10.7202/1006725ar (consulté le 20/07/2020)

Bouchardon Serge, « Towards Gestural Specificity in Digital Literature", electronic book review [En ligne], 12 février 2018. URL : https://electronicbookreview.com/essay/towards-gesturalspecificity-in-digital-literature/ (consulté le 20/07/2020) 
Cayatte Rémi, « Temps de la chose-racontée et temps du récit vidéoludique : comment le jeu vidéo raconte? ", Sciences du jeu [en ligne], nº 9, 2018. URL: http://journals.openedition.org/sdj/ 936 (consulté le 20/07/2020)

Cayatte Rémi, «La narration procédurale au service de la transmission d'émotions : (s')exprimer par le jeu », colloque Lusor in Fabula, Université de Rouen, 8-9 novembre 2019.

Chapman Owen et Sawchuck Kim, « Research-Creation: Intervention, Analysis and "Family Resemblances"», Canadian Journal of Communication, n³7, 2012.

Chauvin Simon, Levieux Guillaume, Donnart Jean-Yves et Natkin Stéphane, « An Out-OfCharacter Approach to Emergent Games Narratives », Proceedings of the 9th International Conference on the Foundations of Digital Games, 2014. URL : https://hal.archives-ouvertes.fr/ hal-01126515 (consulté le 20/07/2020)

Despain Wendy et Ash Lauryn, « Designing for ludonarrative harmony », [En ligne], 2016. URL : https://www.academia.edu/34283487/Designing_For_Ludonarrative_Harmony (consulté le 20/07/2020)

Dubbleman Teun, « Narrative Game Mechanics », in Frank Nack, Andrew S. Gordon (dir.), Proceedings of the 9th International Conference on Interactive Digital Storytelling, Cham : Springer, 2016.

Dupont Bruno, « Le rythme vidéoludique comme mouvement : Second Sight et la ritournelle », Interval(le)s, $n^{\circ} 7,2015$. URL : https://orbi.uliege.be/bitstream/2268/177889/1/ dupont\%202015.pdf (20/07/2020)

Dunne Daniel, «Multimodality or Ludo-Narrative Dissonance », in Nesbitt, K., Blackmore, K. et Smith, S.P. (dir.), Proceedings of the 2014 Conference on Interactive Entertainment, New York : ACM Press, 2014. pp. 1-4.

Fantoli Daria, Heinz Daniel et Wetzel Dennis, « Ludonarrative Dissonance and Gamification: A Systematic Literature Review », séminaire Emerging Trends in Critical Information Infrastructures, juillet 2019. URL : https://www.researchgate.net/publication/ 339384235_Ludonarrative_Dissonance_and_Gamification_A_Systematic_Literature_Review (consulté le 20/07/2020)

Fludernik Monika, Towards a 'natural' narratology, London; New York: Routledge, 1996.

Genette Gérard, Figures II, Paris : Seuil, 1969.

Genvo Sébastien, «L'art du game design : caractéristiques de l'expression vidéoludique », Colloque « E-formes 2 : les arts numériques au risque du jeu », Saint-Étienne, 2008.

Genvo Sébastien, « Comprendre et développer le potentiel expressif », Hermès, La Revue, vol. 62, $\mathrm{n}^{\circ}$ 1, 2012, p. 127-133.

Giner Esteban, « Pour une théorie radicale et dynamique des récits vidéoludiques : la narration à n-corps », colloque Lusor in Fabula, Université de Rouen, 8-9 novembre 2019.

Gosselin Pierre, « La recherche en pratique artistique », dans La recherche création. Pour une compréhension de la recherche en pratique artistique, Gosselin Pierre et Le coquiec Eric (dir.), Québec : Presses de l'Université du Québec, 2006.

Hansen Damien, « Morphologie du jeu vidéo : le ludème envisagé comme unité minimale fonctionnelle du jeu », colloque Les langages du jeu vidéo : codes, discours et images en jeu, Université de Lausanne, 24 au 26 octobre 2019. URL : https://www.youtube.com/watch? v=oATGRysLLik (consulté le 20/07/2020) 
Heussner Tobias, Finley Toiya, Lemay Ann, Brandes Helper Jennifer, The Game Narrative Toolbox, Abington : Routledge, 2015.

Heussner Tobias (dir.), The Advanced Game Narrative Toolbox, Boca Raton, CRC Press, 2019.

Hocking Clint, « Ludo-narrative dissonance in Bioshock », click nothing. Design from a long time ago [en ligne], 7 octobre 2007. URL : https://clicknothing.typepad.com/click_nothing/2007/10/ ludonarrative-d.html (consulté le 20/07/2020).

Järvinen Aki, Games without Frontiers: Theories and Methods for Game Studies and Design, thèse de doctorat, Tampere University, 2008.

Järvinen Aki, « Clickability: a design concept for social games », Gamasutra [En ligne], 3 avril 2010. URL : https://www.gamasutra.com/blogs/AkiJarvinen/20100304/86806/

Clickability_A_Design_Concept_for_Social_Games.php (consulté le 20/07/2020)

Jenkins Henri, « Game Design as Narrative Architecture Architecture », in Salen Katie et Eric Zimmerman (dir.), The Game Design Reader: A Rules of Play Anthology, Cambridge, The MIT Press, 2006, pp. 670-689.

Krichane Sélim, «L'intrigue en trois dimensions », Cahiers de Narratologie [en ligne], n 27, 2014. URL : http://journals.openedition.org/narratologie/7014 (consulté le 20/07/2020)

Larsen Bjarke et Shoenau-Fog Henrik, « The Narrative Quality of Game Mechanics », in Frank Nack, Andrew S. Gordon (dir.), Proceedings of the 9th International Conference on Interactive Digital Storytelling, Cham : Springer, 2016.

Louchart Sandy, Aylett Ruth, Dias Joäo et Figureiredo Rui, « Authoring Emergent narrative-based games », [En ligne], 2008. URL : https://www.researchgate.net/publication/ 228361928_Authoring_emergent_narrative-based_games (consulté le 20/07/2020)

Makedonski Brett, « Ludonarrative Dissonance: Roadblock to realism », Destructoïd [En ligne], 26 septembre 2012. URL : https://www.destructoid.com/stories/ludonarrative-dissonance-theroadblock-to-

realism-235197.phtml\#: :text=At\%20its\%20core\%2C\%20ludonarrative\%20dissonance,the\%20experience\%20to\%20a\%20degree (consultée le 20/07/2020)

Mauger Vincent, « Design narratif : considérations préalables à son étude et à l'analyse de compositions ludofictionnelles sous le modèle EST », Sciences du jeu [En ligne], $n^{\circ} 9,2018$. URL : http://journals.openedition.org/sdj/985 (consulté le 20/07/2020)

Morisset Thomas, «Les jeux vidéo ou la dépense des images », Colloque Jeu vidéo : singularités d'un art de l'écran, Université de Strasbourg, 9 avril 2015.

Posey Jay, « Narrative Design », in Despain Wendy (dir.), Professional Techniques for Video Game Writing, A K Peters/CRC Press, 2008.Neitzel Britta, « Narrativity in Computer Games », in Raessens Joost et Jeffrey Goldstein (dir.), Handbook of Computer Game Studies, Cambridge, The MIT Press, 2005, pp. 227-245.

Paquin Louis-Claude et Noury Cynthia, Cartographies de la recherche-création, lcpaquin.com [en ligne], 2018. URL : http://lcpaquin.com/cartoRC/ (consulté le 20/07/2020)

Papale Luca, « Beyond identification: defining the relationships between player and avatar ", Journal of Games criticism [En ligne], 2014. URL : http://gamescriticism.or g/articles/papale-1-2/ (consulté le 20/07/2020)

Patron Sylvie (dir.) Introduction à la narratologie postclassique. Les nouvelles directions de la recherche sur le récit, Villeneuve d'Ascq, Presses Universitaires du Septentrion, 2017. 
Prince Gérald, « Narratologie classique et narratologie post-classique », Vox Poetica [En ligne], 2006. URL : http://www.vox-poetica.org/t/articles/prince.html (consulté le 20/07/2020)

Portnow James (Extracredits), « Mechanics as Metaphor: How gameplay itself tells a story », Youtube [En ligne], 22 août 2012. URL : https://www.youtube.com/watch?v=4QwcI4iQt2Y (consulté le 20/07/2020)

Ryan Marie-Laure, « Cognitive Maps and the Construction of Narrative Space », in David Herman (dir), Narrative Theory and the Cognitive Sciences. Stanford : CSLI, 2003, pp. 214-242.

Ryan Marie-Laure, Avatars of Story, Minneapolis, University of Minnesota Press, 2006.

Ryan Marie-Laure et Thon Jan-Noel, « Introduction », in Storyworlds across Media, Marie-Laure Ryan et Jan-Noel Thon (dir.), Lincoln : University of Nebraska Press, 2014.

Séraphine Frédéric, « Ludonarrative dissonance: Is storytelling about reaching harmony?», fredericseraphine.com, 2 septembre 2016. URL : http://www.fredericseraphine.com/index.php/ 2016/09/02/ludonarrative-dissonance-is-storytelling-about-reaching-harmony/ (consulté le 20/07/2020)

Short Emily, Emily Short's Interactive Storytelling [En ligne]. URL : https://emshort.blog/ (consulté le 20/07/2020).

Sicart Miguel, « Defining game mechanics », Game Studies [En ligne], vol. 8, n² 2, 2008.

Sicart Miguel, « Against Procedurality », Game Studies [En ligne], vol. 11, n 3, 2011. URL : http:// gamestudies.org/1103/articles/sicart_ap (consulté le 20/07/2020)

Sicart Miguel, « Papers, please. Ethics. », in Matthew Thomas Payne, Nina B. Huntemann (dir.), How to Play Video Games, New York : NYU Press, 2019.

URL : http://gamestudies.org/0802/articles/sicart (consulté le 20/07/2020)

Triclot Matthieu, Philosophie des jeux vidéo, Paris : Zone, 2011.

Triclot Matthieu, «Les jeux vidéo en aveugle : essai de rythmanalyses », in K. Langewiesche \& J-B. Ouédraogo (dir.), L'enquête et ses graphies en sciences sociales : figurations iconographiques d'après société, Amalion, 2019, pp. 175-194.

Walon Sophie, «Les corporéités de la danse contemporaine française expérimentale : une pratique philosophique et politique de "résistance" ", Agôn [En ligne], 2011. URL : https:// journals.openedition.org/agon/1927 (consulté le 20/07/2020)

Wardrip-Fruin Noah, Expressive Processing. Digital Fictions, Computer Games and Software Studies, Cambridge \& London: The MIT Press, 2009.

Wiemin Toh, A multimodal discourse of analysis of video games. A ludonarrative model, thèse de doctorat, National University of Singapore, 2015

ZABBAN Vinciane, «Retour sur les game studies. Comprendre et dépasser les approches formelles et culturelles du jeu vidéo ", Réseaux [En ligne], $\mathrm{n}^{\circ}$ 173-174, 2012. URL : https:// www.cairn.info/revue-reseaux-2012-3-page-137.htm (consulté le 20/07/2020).

\section{NOTES}

1. Vinciane ZABBAN, «Retour sur les game studies. Comprendre et dépasser les approches formelles et culturelles du jeu vidéo ", Réseaux [En ligne], $n^{\circ}$ 173-174, 2012. URL : https:// www.cairn.info/revue-reseaux-2012-3-page-137.htm (consulté le 20/07/2020). 
2. Britta Neitzel, « Narrativity in Computer Games », in Raessens Joost et Jeffrey Goldstein (dir.), Handbook of Computer Game Studies, Cambridge, The MIT Press, 2005, pp. 227-245.

3. Marie-Laure Ryan, Avatars of Story, Minneapolis, University of Minnesota Press, 2006.

4. Dominic Arsenault, «Jeux et enjeux du récit vidéoludique : la narration dans le jeu vidéo » [En ligne], Mémoire de master en études cinématographiques, sous la direction de Bernard Perron, université de Montréal, 2006. URL: http://www.le-ludophile.com/Files/ Dominic\%20Arsenault\%20-\%20Memoire.pdf (consulté le 20/07/2020)

5. Ibid, p. 79.

6. Rémi Cayatte, «Temps de la chose-racontée et temps du récit vidéoludique : comment le jeu vidéo raconte?", Sciences du jeu [En ligne], n 9, 2018. URL: http://journals.openedition.org/sdj/ 936 (consulté le 20/07/2020)

7. Sélim Krichane, "L’intrigue en trois dimensions », Cahiers de Narratologie [En ligne], $\mathrm{n}^{\circ}$ 27, 2014. URL : http://journals.openedition.org/narratologie/7014 (consulté le 20/07/2020)

8. Henri Jenkins, "Game Design as Narrative Architecture Architecture », in Salen Katie et Eric Zimmerman (dir.), The Game Design Reader: A Rules of Play Anthology, Cambridge, The MIT Press, 2006, pp. 670-689.

9. Fanny Barnabé, Narration et jeu vidéo. Pour une exploration des univers fictionnels, mémoire de Master, sous la direction de Bjorn-Olav Dozo, université de Liège, 2012.

10. Sébastien Genvo, "Comprendre et développer le potentiel expressif ", Hermès, La Revue, vol. 62, n 1, 2012, pp. 127-133.

11. Sébastien Genvo, "L'art du game design : caractéristiques de l'expression vidéoludique ", Colloque «E-formes 2 : les arts numériques au risque du jeu », Saint-Étienne, 2008.

12. Vincent Mauger, «Design narratif : considérations préalables à son étude et à l'analyse de compositions ludofictionnelles sous le modèle EST », Sciences du jeu [En ligne], $n^{\circ} 9,2018$. URL : http://journals.openedition.org/sdj/985 (consulté le 20/07/2020)

13. Jay Posey, "Narrative Design ", in Despain Wendy (dir.), Professional Techniques for Video Game Writing, A K Peters/CRC Press, 2008, p. 55. ["if narrative is "the story our game tells," narrative design is the creation of that story and the design of the mechanics through which it is told. That is, narrative design encompasses not only the story itself but also how the story is communicated to players and how other game features support and immerse the player within the game world."]

Les traductions dans cet article sont le fait de l'auteure.

14. Tobias Heussner, Toiya Finley, Ann Lemay, Jennifer Brandes Helper, The Game Narrative Toolbox, Abington : Routledge, 2015, p. 8. ["A Game Narrative Designer is the champion of an interactive story [...] and combines the roles of a writer with that of a game designer."]

15. Ibid, p.17. ["Game Narrative Design is the art of telling a story in a computer game using the techniques and devices available. It is the art of using gameplay and the sum of visual and acoustic methods to create an entertaining and engaging experience for players."]

16. Rémi Cayatte, «La narration procédurale au service de la transmission d'émotions : (s')exprimer par le jeu », colloque Lusor in Fabula, Université de Rouen, 8-9 novembre 2019.

17. Emily Short, Emily Short's Interactive Storytelling [En ligne]. URL : https://emshort.blog/ (consulté le 20/07/2020).

18. Heussner Tobias (dir.), The Advanced Game Narrative Toolbox, Boca Raton, CRC Press, 2019.

19. Esteban Giner, «Pour une théorie radicale et dynamique des récits vidéoludiques : la narration à n-corps », colloque Lusor in Fabula, Université de Rouen, 8-9 novembre 2019.

20. Comme celles de :

Gerard Genette, Figures II, Paris : Seuil, 1969. 
Rene Audet, «La narrativité est affaire d'événement », in Jeux et enjeux de la narrativité dans les pratiques contemporaines, Rene Audet et al., Paris : Dis Voir, 2006.

21. Marie-Laure Ryan et Jan-Noel Thon, "Introduction ", in Storyworlds across Media, Marie-Laure Ryan et Jan-Noel Thon (dir.), Lincoln : University of Nebraska Press, 2014.

22. Gérald Prince, « Narratologie classique et narratologie post-classique », Vox Poetica [En ligne], 2006. URL : http://www.vox-poetica.org/t/articles/prince.html (consulté le 20/07/2020)

23. Sylvie Patron (dir.) Introduction à la narratologie postclassique. Les nouvelles directions de la recherche sur le récit, Villeneuve d'Ascq, Presses Universitaires du Septentrion, 2017.

24. Rapahël Baroni, Les Rouages de l'intrigue. Les outils de la narratologie postclassique pour l'analyse des textes littéraires, Genève, Slatkine, 2017.

25. Aki Järvinen, Games without Frontiers: Theories and Methods for Game Studies and Design, thèse de doctorat, Tampere University, 2008.

26. Miguel Sicart, « Defining game mechanics », Game Studies [En ligne], vol. 8, $n^{\circ} 2$, 2008. URL : http://gamestudies.org/0802/articles/sicart (consulté le 20/07/2020)

27. Damien Hansen, «Morphologie du jeu vidéo : le ludème envisagé comme unité minimale fonctionnelle du jeu ", colloque Les langages du jeu vidéo : codes, discours et images en jeu, Université de Lausanne, 24 au 26 octobre 2019. URL: https://www.youtube.com/watch?v=oATGRysLLik (consulté le 20/07/2020)

28. Ibid.

29. Pierre Gosselin, « La recherche en pratique artistique », dans La recherche création. Pour une compréhension de la recherche en pratique artistique, Gosselin Pierre et Le coquiec Eric (dir.), Québec : Presses de l'Université du Québec, 2006. / Louis-Claude Paquin et Cynthia Noury, Cartographies de la recherche-création, lcpaquin.com [En ligne], 2018. URL : http://lcpaquin.com/cartoRC/ (consulté le 20/07/2020)

30. Owen Chapman et Kim Sawchuck, « Research-Creation: Intervention, Analysis and "Family Resemblances"», Canadian Journal of Communication, n 37, 2012.

31. Le Game Design du projet a été conçu par Chabane Hadji, Jérôme Tsiné et Lucas Abadie, fondateurs du studio.

32. Clint Hocking, «Ludo-narrative dissonance in Bioshock », click nothing. Design from a long time ago [En ligne], 7 octobre 2007. URL : https://clicknothing.typepad.com/click_nothing/2007/10/ ludonarrative-d.html (consulté le 20/07/2020).

33. Ibid. ["Bioshock seems to suffer from a powerful dissonance between what it is about as a game, and what it is about as a story"]

34. Daniel Dunne, «Multimodality or Ludo-Narrative Dissonance », in Nesbitt, K., Blackmore, K. et Smith, S.P. (dir.), Proceedings of the 2014 Conference on Interactive Entertainment, New York : ACM Press, 2014. pp. 1-4.

35. Fanny Barnabé et Julie Delbouille, « Aux frontières de la fiction : l'avatar comme opérateur de réflexivité », Sciences du jeu [En ligne], no 9, 2018. URL : http://journals.openedition.org/sdj/ 958 (consulté le 20/07/2020)

36. Une revue plus complète de la littérature critique peut être consultée ici :

Daria Fantoli, Daniel Heinz et Dennis Wetzel, «Ludonarrative Dissonance and Gamification: A Systematic Literature Review » (séminaire Emerging Trends in Critical Information Infrastructures, juillet 2019. URL : https://www.researchgate.net/publication/ 339384235_Ludonarrative_Dissonance_and_Gamification_A_Systematic_Literature_Review (consulté le 20/07/2020)

37. Brett Makedonski, «Ludonarrative Dissonance: Roadblock to realism », Destructoïd [En ligne], 26 septembre 2012. URL : https://www.destructoid.com/stories/ludonarrative-dissonance-theroadblock-torealism-235197.phtml\#: :text=At\%20its\%20core\%2C\%20ludonarrative\%20dissonance,the\%20experience\%20to\%20a\%20degree 
(consultée le 20/07/2020) ["At its core, ludonarrative dissonance is the idea that when a game tells the player one thing through its story and environment, and then contradicts it though gameplay, the player becomes unimmersed and disconnected from the experience to a degree."] 38. Monika Fludernik, Towards a 'natural' narratology, London; New York: Routledge, 1996.

39. Marie-Laure Ryan, " Cognitive Maps and the Construction of Narrative Space ", in David Herman (dir), Narrative Theory and the Cognitive Sciences. Stanford : CSLI, 2003, pp. 214-242.

40. Toh Wiemin, A multimodal discourse of analysis of video games. A ludonarrative model, thèse de doctorat, National University of Singapore, 2015, p. 306. ["Ludonarrative resonance" occurs when the narrative and gameplay concepts are so integrated with each other that they cannot be separated. When this occurs, the players feel a heightened emotional experience [...]"]

41. Wendy Despain et Lauryn Ash, « Designing for ludonarrative harmony », [En ligne], 2016. URL : https://www.academia.edu/34283487/Designing_For_Ludonarrative_Harmony (consulté le 20/07/2020)

42. A ce sujet, on pourra notamment consulter : Grésy Brigitte et Becker Marie, Le sexisme dans le monde du travail. Entre déni et réalité. Rapport du Conseil supérieur de l'égalité professionnelle entre les femmes et les hommes n²015-01 publié le 6 mars 2015. URL : https://www.egalitefemmes-hommes.gouv.fr/wp-content/uploads/2015/03/RAPPORT-CSEP-V7BAT.pdf

43. Voir: Fanny Barnabé et Julie Delbouille, «Aux frontières de la fiction: l'avatar comme opérateur de réflexivité ", art.cit.

44. Nick Ballantyne, "The Why, What and WTF: Ludonarrative Dissonance ", Gamecloud [En ligne], 15 février 2015. URL : https://gamecloud.net.au/features/opinion/twwwtf-ludonarrativedissonance (consulté le 20/07/2020)

45. Ibid. ["if we are not given the allowance to change our play-style or ignore the contradictions, we're only left to justify the dissonance"]

46. Ibid. ["the sensation of being pulled out of the play experience."]

47. Voir : Luca Papale, «Beyond identification : defining the relationships between player and avatar", Journal of Games criticism [En ligne], 2014. URL : http:// gamescriticism.or g/articles/papale-1-2/ (consulté le 20/07/2020)

48. Frédéric Séraphine, «Ludonarrative dissonance: Is storytelling about reaching harmony? ", fredericseraphine.com, 2 septembre 2016. URL : http://www.fredericseraphine.com/index.php/ 2016/09/02/ludonarrative-dissonance-is-storytelling-about-reaching-harmony/ (consulté le 20/07/2020) ["the annihilation of any form of pre-built narrative structure"]

49. Henri Jenkins, " Game Design as Narrative Architecture Architecture », art. cit.

50. Sandy Louchart, Ruth Aylett, Joäo Dias et Rui Figureiredo, « Authoring Emergent narrativebased games », [En ligne], 2008. URL : https://www.researchgate.net/publication/ 228361928_Authoring_emergent_narrative-based_games (consulté le 20/07/2020)

51. Simon Chauvin, Guillaume Levieux, Jean-Yves Donnart et Stephane Natkin, «An Out-OfCharacter Approach to Emergent Games Narratives ", Proceedings of the 9th International Conference on the Foundations of Digital Games, 2014. URL : https://hal.archives-ouvertes.fr/hal-01126515 (consulté le 20/07/2020)

52. Teun Dubbleman, «Narrative Game Mechanics », in Frank Nack, Andrew S. Gordon (dir.), Proceedings of the 9th International Conference on Interactive Digital Storytelling, Cham : Springer, 2016. ["Narrative game mechanics invite agents, including the player, to perform actions that support the construction of engaging stories and fictional worlds in the embodied mind of the player."]

53. Ibid. ["Depending on how the player chooses to engage with the mechanics and rules, any of these stories could unfold."]

54. On peut citer par exemple: Miguel Sicart, "Papers, please. Ethics. ", in Matthew Thomas Payne, Nina B. Huntemann (dir.), How to Play Video Games, New York : NYU Press, 2019. 
55. Bjarke Larsen et Henrik Shoenau-Fog, "The Narrative Quality of Game Mechanics », in Frank Nack, Andrew S. Gordon (dir.), Proceedings of the 9th International Conference on Interactive Digital Storytelling, Cham : Springer, 2016.

56. Ibid. ["Through the mechanics, the players themselves get to 'feel' the narrative and experience their own version of the narrative that shines through the gameplay and the context"]

57. Ibid. ["game mechanics inherently convey a narrative through the possibilities and valorization of actions given to the player"]

58. James Portnow (Extracredits), "Mechanics as Metaphor: How gameplay itself tells a story », Youtube [En ligne], 22 août 2012. URL: https://www.youtube.com/watch?v=4QwcI4iQt2Y (consulté le 20/07/2020)

59. Aki Järvinen, Games without Frontiers: Theories and Methods for Game Studies and Design, op.cit.

60. Anna Anthropy et Naomi Clark, Game Design Vocabulary : Exploring the Fundational Principles Behind Good Game Design, Boston: Addison-Wesley Professional, 2014, p. 171. ["The Best Amendment doesn't explain its message with words, but in authoring a system that produces certain kinds of experiences when played, Pedercini gets an idea across: who's a "good guy with a gun" depends on your perceptions, and running around shooting apparent "bad guys" leads to a general bloodbath."]

61. Emily Short, « The Anatomy of a Story (John Truby)», Emily Short's Interactive Storytelling [En ligne], 2 avril 2019. URL: https://emshort.blog/2019/04/02/the-anatomy-of-a-story-john-truby/ (consulté le 20/07/2020) ["the preparation to find a good mechanic for your narrative design"] 62. Frédéric Séraphine, «Ludonarrative dissonance: Is storytelling about reaching harmony? ", art. cit. ["changing a game mechanics or making it disappear will sometimes have the ability to create meaning about the game world or its characters."]

63. Ian Bogost, Persuasive Games, Cambridge \& London: The MIT Press, 2007.

64. Noah Wardrip-Fruin, Expressive Processing. Digital Fictions, Computer Games and Software Studies, Cambridge \& London: The MIT Press, 2009, p. 4. ["what processes express in their design-which may not be visible to audiences"]

65. Miguel Sicart, « Against Procedurality », Game Studies [En ligne], vol. 11, n 3, 2011. URL : http://gamestudies.org/1103/articles/sicart_ap (consulté le 20/07/2020)

66. Thomas Morisset, «Les jeux vidéo ou la dépense des images ", Colloque Jeu vidéo : singularités d'un art de l'écran, Université de Strasbourg, 9 avril 2015.

67. Serge Bouchardon, «Des figures de manipulation dans la création numérique ", Protée, vol. 39, nº 1, 2011, pp. 37-46. URL : https://doi.org/10.7202/1006725ar (consulté le 20/07/2020)

68. Aki Järvinen, «Clickability: a design concept for social games », Gamasutra [En ligne], 3 avril 2010. URL : https://www.gamasutra.com/blogs/AkiJarvinen/20100304/86806/ Clickability_A_Design_Concept_for_Social_Games.php (consulté le 20/07/2020)

69. Bruno Dupont, «Le rythme vidéoludique comme mouvement : Second Sight et la ritournelle », Interval(le)s, $\mathrm{n}^{\circ} 7$, 2015. URL : https://orbi.uliege.be/bitstream/2268/177889/1/ dupont\%202015.pdf (20/07/2020)

70. Serge Bouchardon, «Towards Gestural Specificity in Digital Literature”, electronic book review [En ligne], 12 février 2018. URL : https://electronicbookreview.com/essay/towards-gesturalspecificity-in-digital-literature/ (consulté le 20/07/2020) ["with the Digital, the interactive gesture and the interactive gestural manipulation are defamiliarized thanks to the opacity of computation: the Digital can introduce a gap between the user's expectations based on his/her gestures and the realm of possibilities offered."]

71. Sophie Walon, «Les corporéités de la danse contemporaine française expérimentale : une pratique philosophique et politique de "résistance" ", Agôn [En ligne], 2011. URL : http:// agon.ens-lyon.fr/index.php?id=1927 (consulté le 20/07/2020) 
72. Matthieu Triclot, «Les jeux vidéo en aveugle : essai de rythmanalyses », in K. Langewiesche \& J-B. Ouédraogo (dir.), L'enquête et ses graphies en sciences sociales : figurations iconographiques d'après société, Amalion, 2019, pp. 175-194.

73. Matthieu Triclot, Philosophie des jeux vidéo, Paris : Zone, 2011.

\section{ABSTRACTS}

Anchored in contemporary scientific research about video games narrativity and tackling current considerations in the field of Narrative Design, his paper presents a research-creation project that explores the idea of a narrative value of the game mechanics experienced through the body. More precisely, three concepts are discussed: the ludo-narrative dissonance and harmony, the narrative game mechanics and the narrative body experience of the game.

Faisant écho aux recherches contemporaines sur la narrativité du jeu vidéo et s'inscrivant au cœur des problématiques du Narrative Design, cet article s'appuie sur un travail de recherchecréation pour défendre l'idée d'une valeur narrative de l'expérience corporelle des mécaniques ludiques. Il met plus précisément en lumière les potentiels expressifs de la dissonance et de l'harmonie ludo-narrative, la portée narrative des mécaniques ludiques et l'importance de la corporéité dans la construction du sens de l'expérience.

\section{INDEX}

Geographical index: Europe, Etats-Unis, Canada, Japon Chronological index: XXIe siècle

Keywords: Game Studies, Play Studies, dissonance ludo-narrative

Mots-clés: Narrative Design, narrativité vidéoludique, recherche-création

\section{AUTHOR}

\section{HÉLÈNE SELLIER}

Docteure en littératures comparées, Membre de l'Observatoire des mondes numériques en sciences humaines [OMNSH], Membre de l'association Littératures populaires et cultures médiatiques [LPCM], Chargée de recherches au sein du studio de jeux vidéo The Seed Crew 